past medical history. Family history showed breast and ovarian cancer in $104(48.1 \%)$ and 49 patients (22.7\%). BRCA1 mutation was underlying in $121(56.0 \%)$ patients, whereas 64 (29.6\%) patients had a BRCA2 mutation, 22 women $(10.2 \%)$ an unknown mutation status. Nine patients (4.2\%) presented with a RAD51C, Lynch gene, PALB2, or BRIP1 mutation. Most patients (99.1\%) underwent minimal invasive surgery. Incidental findings of a STIC lesion and/or ovarian cancer was detected in $7(3.2 \%)$ and $5(2.3 \%)$ patients. Median age of those 12 women was 56 years (range 44-79) and all underwent a staging surgery. Final histopathological results showed high grade serous ovarian cancer in 5 patients: FIGO IA $(n=1)$, FIGO IB $(n=1)$, FIGO IC $(n=1)$, FIGO IIA $(n=1)$, FIGO IIIC $(n=1)$. Seven women had STIC lesions. All patients with ovarian cancer had a BRCA1 mutation and one patient even had a BRCA1 and RAD51C mutation. Of all STIC patients five (71.4\%) had a BRCA1 and two (28.6\%) had a RAD51C mutation.

Conclusion Our results of incidental STIC/ovarian cancer findings confirm the results published in the literature. RRSO is highly recommended in mutation carriers. Genetic counselling and testing need to be routinely offered to family members of mutation gene carriers. It is from tremendous importance to raise awareness that in women at high risk, the development of premalignant neoplasms and malignancies can be prevented. Timing of RRSO should be independent of age and promptly after completion of family planning.

Disclosures SE received non-financial support from Tesaro, outside the submitted work.

JPR has no conflict of interest to declare.

$\mathrm{AdB}$ reports personal fees from Roche, Astra Zeneca, Tesaro, Clovis, Pfizer, Genmab, Pharmar, and Biocad, outside the submitted work.

$\mathrm{PH}$ reports grants and personal fees from Astra Zeneca and Roche, personal fees from Sotio, grants and personal fees from Tesaro and GSK, personal fees from Stryker,Zai Lab, and MSD, grants from Public funding (DKH, DFG, EU), personal fees from Clovis, andImmunogen, grants from Boehringer Ingelheim, Medac, and Genmab, outside the submitted work.

SS reports personal fees from Roche, Astra Zeneca, personal fees and travel grants from GSK/Tesaro, personal fees from Clovis and travel grants from Pharmamar, outside the submitted work.

TB has been an advisor for Tesaro and received research grant from Amgen, non-financial support from Amgen, MSD, Roche, and Tesaro, outside the submitted work.

AT, SH, NP have no conflicts of interest do declare.

BA reports personal fees and non-financial support from Roche, personal fees from Amgen and Astra Zeneca, personal fees and non-financial support from Tesaro, personal fees from Clovis and Celgene, non-financial support from PharmaMar, outside the submitted work.

\section{EPIDEMIOLOGY OF OVARIAN CANCER IN KAZAKHSTAN (2013-2018)}

Dilyara Kaidarova, Yerlan Kukubassov, Raikhan Bolatbekova, Alima Satanova. Kazakh Institute of Oncology and Radiology; Oncogynecology
Introduction/Background Worldwide, ovarian cancer (OC) is the seventh most common cancer in women, with a fiveyear survival rate below $45 \%$. Every year around the world, OC is diagnosed in 240,000 women. Studies on the epidemiology of OC were carried out in different regions of the world, taking into account various factors. At the same time, the issues of the relationship between morbidity and mortality from OC with genetic, hormonal factors, as well as nutritional factors, morphometric factors, somatic pathology, socio-demographic and other factors were taken into account.

The problem of OC epidemiology is extremely relevant for the Republic of Kazakhstan due to the significant prevalence of this disease among the female population, the still high level of neglected cases, as well as high mortality.

Methodology To analyze the epidemiological data of OC in the world, materials from the Globocan 2018 database of the International Agency for Research on Cancer (IARC) were used. To analyze the main statistical data for the regions of Kazakhstan, statistical data from the Cancer Register of the Republic of Kazakhstan for 2013-2018 were used.

Results In the Republic of Kazakhstan alone, there are more than 1000 new cases of OC and more than 400 deaths from this disease per year [5], while in the United States there are more than 22,000 new cases of OC and 14,000 deaths per year $[6,7]$.

In Kazakhstan, malignant neoplasms of the ovaries occupies the 3rd rank position among gynecological cancers. When analyzing rough intensive indicators of the incidence of ovarian cancer, there is an increase in the detection rate of this disease for the period from 2013 to 2018.[8-10]

The analysis of age-related incidence rates showed that malignant neoplasms of the ovaries are found in all age groups, with a noticeable increase by 65-69 years. The main contingent of the sick are women of working age. Also, when analyzing this five-year period (2013-2018), there is a decrease in the incidence in childhood and adolescence, so in 2014, 5 cases of ovarian cancer were recorded in the age group 5-19 years, and in 2019 - 1 case of this disease. Over the past decade, there has been an increase in morbidity at the age of 55-65 years [5].

Conclusion Morbidity and mortality from OC remain an urgent epidemiological problem in Kazakhstan and require further scientific research to identify risk factors. There are regions in the Republic of Kazakhstan that exceed the national average. In these regions, it is necessary to more widely apply modern methods of early diagnosis and treatment of ovarian cancer.

If detected at earlier stages, it is possible to obtain significant results of OC treatment. The main tasks of OC epidemiology are: continuation of in-depth studies of the prevalence in the regions of the Republic of Kazakhstan with the identification of population groups and regions with the lowest and highest rates of morbidity and mortality from OC.

Disclosures Epidemiological data on the incidence rates of malignant neoplasms of the ovaries according to Globocan 2018 show significant differences across countries (per 100,000 women): from 3.8 in Central Africa to 11.9 in Central and Eastern Europe [1]. 